\title{
NIVEL DE CONOCIMIENTO/CARACTERÍSTICAS SOCIODEMOGRÁFICAS DE LAS ESTUDIANTES UNIVERSITARIAS QUE UTILIZAN ANTICONCEPTIVOS ORALES DE EMERGENCIA
}

\section{Level of Knowledge / Demographic Characteristics of College Students that use Emergency Oral Contraception}

\author{
*José Raúl Arita Chávez, **Cinea Mabeth Abujieres Galeano, \\ **Verónica Eunice Cruz Aguilar.
}

\section{RESUMEN}

El gran auge alcanzado por los anticonceptivos orales de emergencia (AOE) en los últimos años se ha convertido en un tema de interés social. Objetivo: Evaluar el nivel de conocimiento y las características sociodemográficas de las estudiantes universitarias que utilizan anticonceptivos orales de emergencia. Pacientes y Métodos: Estudio descriptivo-transversal, realizado en la Universidad Nacional Autónoma de Honduras en el Valle de Sula (UNAH-VS), en el tercer trimestre del año 2014. El muestreo fue por conveniencia, incluyendo a 66 estudiantes que usaban $\mathrm{AOE}$. La información se obtuvo mediante un instrumento de recolección de datos tipo cuestionario. Resultados: Se encontró que el nivel de conocimiento respecto a los AOE era bajo en 32(48.5\%) estudiantes. Las características sociodemográficas predominantes en dichas estudiantes fueron: Edad 17 y 20 años 33(50.0\%), procedentes del área urbana $51(77.3 \%)$, residentes en San Pedro Sula 42(63.6\%), ingreso familiar entre Lps. 8,000-16,000 $32(48.5 \%), 41(62.1 \%)$ pertenecía a las carreras de las ciencias sociales y las ciencias biológicas y de la salud, solteras

\footnotetext{
*Médico y Cirujano, Máster en Educación Superior. Jefe de la Escuela Universitaria de Ciencias de la Salud UNAH-VS.

**Estudiantes de V año de la Carrera de Medicina UNAH-VS.

Dirigir correspondencia a: abujieres92@yahoo.com

Recibido: 19 de Marzo 2,015 Aprobado 25 de Julio 2,015
}

$55(83.3 \%)$, no trabajaban $48(72.7 \%)$, y forman parte de la religión católica 31 (47.0\%). Conclusiones: Aproximadamente la mitad de las estudiantes universitarias que utilizan $\mathrm{AOE}$, lo hacen sin tener conocimientos adecuados respecto al tema, poniendo en riesgo su salud.

\section{PALABRAS CLAVE}

Anticonceptivo, Conocimiento, Femenina.

\section{ABSTRACT}

The great boom reached by emergency contraceptive pills (ECPs) in recent years has become a subject of public interest. Objective: To assess the level of knowledge and sociodemographic characteristics of university students using emergency contraceptive pills. Patients and Methodology: Descriptive cross-sectional study, conducted at the National Autonomous University of Honduras in the Valle de Sula (UNAH-VS) in the third quarter of 2014. The convenience sample, including 66 students who used ECPs. The information was obtained by a collection instrument type questionnaire data. Results: We found that the level of knowledge about the AOE was low in 32 (48.5\%) students. Predominant sociodemographic characteristics in these students were: age 17 to 20 years $33(50.0 \%)$, from the urban area $51(77.3 \%)$, living in San 
Pedro Sula 42 (63.6\%), family income between Lps. 8.000 to 16.00032 (48.5\%), 41 $(62.1 \%)$ belonged to the careers of the social sciences and life sciences and health, unmarried 55 (83.3\%), not working $48(72.7 \%)$, and are part of Catholicism 31 (47.0\%). Conclusions: About half of university students using AOE, do not have adequate knowledge on the subject, risking your healt.

\section{KEYWORDS}

Contraceptive, Knowledge, Female.

\section{INTRODUCCIÓN}

La adolescencia suele marcar el inicio de la actividad sexual basada en la erotización de las relaciones. Las relaciones sexuales en los adolescentes y jóvenes están marcadas por la superación de la rigidez y son más abiertas, lo que corresponde a una sociedad más plural. Por otra parte se observan conductas sexuales riesgosas que traen consigo consecuencias como embarazos no deseados, abortos inducidos y muerte materna.

La OMS afirma que de los 210 millones de embarazos anuales en todo el mundo, 80 millones son no planeados, es decir, 2 de cada 5 embarazos. De estos embarazos no planeados, 46 millones (58\%) terminan interrumpiéndose, 19 millones de estos, se dan en países donde la intervención voluntaria del embarazo es ilegal. Dos tercios de los abortos practicados en el año corresponden a mujeres entre 15 a 30 años. En América Latina más del $50 \%$ se practican en mujeres de 20-29 años y casi el $70 \%$ en mujeres menores de 30 años. ${ }^{(1)}$

Estas estadísticas tan abrumadoras contri- buyeron al gran auge alcanzado por los Anticonceptivos Orales de Emergencia (AOE), que se crearon con el fin de disminuir en manera considerable estos índices. Como su nombre lo indica, se utilizan en casos de emergencia, ya sea por fallo del método anticonceptivo habitual o por mantener una relación sexual sin protección por el motivo que fuera. Sin embargo, se ha observado un uso desmedido de estas, tanto así que son utilizadas por muchas jóvenes como anticonceptivos regulares sin tener los conocimientos adecuados.

Los AOE son un método utilizado para el control de la natalidad que previene el embarazo después de una relación sexual sin protección o por el fallo del método habitual. Está hecho a base de levonorgestrel y se le conoce también como "píldora del día después", "píldora de la mañana siguiente" o "plan b". (2) El levonorgestrel es un progestágeno sintético biológicamente activo, que no es efectivo después de las 72 horas; por ser estructuralmente semejante a la progesterona, su mecanismo de acción tiene que ver con el desempeño de esta hormona in vivo. (3) Actúa inhibiendo o retrasando la ovulación, espesando el moco cervical y alterando el movimiento de los espermatozoides en las trompas de Falopio disminuyendo de esta manera la posibilidad de fecundación. ${ }^{(4,5)}$ Es importante recalcar que las píldoras actúan antes de la implantación, por lo tanto, si la implantación ya se ha producido, la gestación es inevitable aunque se tome la píldora.

Los AOE están indicados para prevenir el embarazo después de una relación sexual sin protección y en caso de que falle el 
método anticonceptivo habitual. ${ }^{(4)}$

Los efectos secundarios que se pueden presentar son muy variados, entre estos están: náuseas, vómitos, cefalea, cansancio, mareos, dolor en la parte baja del abdomen, mayor sensibilidad en las mamas, sangrado irregular, la menstruación siguiente puede iniciarse unos cuantos días antes o después de lo normal, trombosis venosa profunda, cáncer de mama y porfiria aguda intermitente. ${ }^{6)}$

El objetivo de la investigación fue evaluar el nivel de conocimiento y las características sociodemográficas de las estudiantes universitarias que utilizan anticonceptivos orales de emergencia.

\section{PACIENTES Y MÉTODOS}

Se realizó un estudio descriptivo, de corte transversal, durante el tercer trimestre del año 2014, el universo estuvo constituido por las 8,525 estudiantes femeninas de la UNAH-VS, se utilizó muestreo por conveniencia; 66 estudiantes que utilizaban AOE.

Se utilizó un instrumento de recolección de datos tipo cuestionario el cual estaba formado por preguntas sociodemográficas y nivel de conocimiento del tema. (Se evaluó el nivel de conocimiento mediante las siguientes preguntas:

1) Funcionarían las pastillas anticonceptivas de emergencia (PAE) si hay un retraso en el período menstrual?

2) Cuanto tiempo después de una relación sexual sin protección, deben tomarse las PAE?

3) Que droga o químico contienen las PAE?

4) Tiene efectos adversos el uso de las
PAE?), se estableció a) Alto, si contestó las cuatro preguntas, b) Medio, si contestaba tres preguntas y c) Bajo, si contestaba dos o una pregunta). La participación fue voluntariamente previo consentimiento informado de forma escrita al inicio del mismo. Los criterios de inclusión fueron: estudiante femenina matriculada en la UNAH-VS durante el tercer período 2014 que refiriera utilizar AOE, que estuviera de acuerdo a participar en la investigación.

Los datos se analizaron mediante PASSW Statistics 18. Inicialmente se aplicó la prueba piloto a 21 estudiantes, obteniendo un alfa de Cronbach de 0.885 . Una vez completadas las encuestas se calcularon las medidas de tendencia central.

\section{RESULTADOS}

Se encontró que $32(48.5 \%)$ estudiantes universitarias que utilizaban $\mathrm{AOE}$, lo hacían teniendo bajo nivel de conocimiento del tema. Ver gráfico $\mathrm{N}^{\circ} 1$.

Grafico $N^{\circ} 1$ : Nivel de Conocimiento sobre AOE de las estudiantes

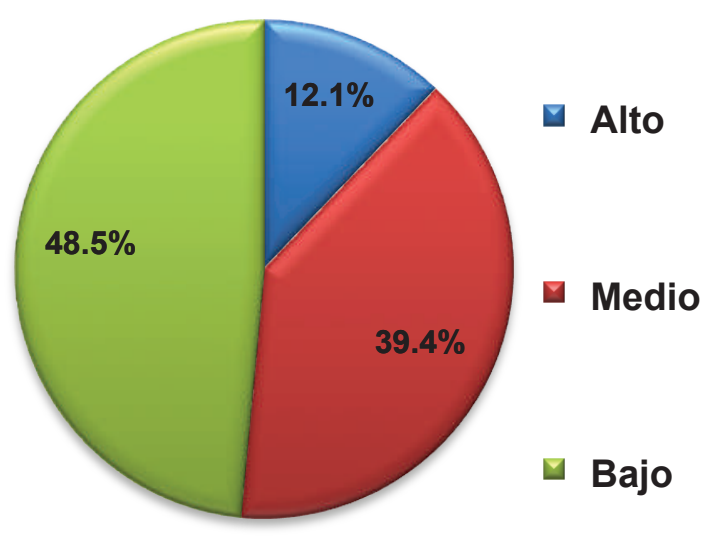

Fuente: Instrumento de recolección de datos 
Las características sociodemográficas fueron:

Edad: $21(31.8 \%)$ tenía entre 17-20 años, 33 (50.0\%) 21-24 años y 12 (18.2\%) 25 o más años. Lugar de Procedencia: 15 (22.7\%) procedían del área rural y 51 (77.3\%) del área urbana. Lugar de Residencia: San Pedro Sula 42 (63.6\%), Choloma 4 (6.1\%), Villanueva 6 (9.1\%), La Lima 6 (9.1\%), El Progreso 5 (7.6\%) y Puerto Cortés $3(4.5 \%)$. Ingreso Económico Familiar: 6 (9.1\%) tenía un ingreso familiar menor a Lps. 8,000, $32(48.5 \%)$ entre Lps. 8,000-16,000 y 28 (42.4\%) un ingreso familiar mayor a Lps. 16,000. Área de estudio: 23 (34.8\%) de las estudiantes universitarias que utilizaban AOE pertenecían al área de las ciencias sociales. (Ver gráfico $\mathrm{N}^{\circ} 2$ ). Estado Civil: En cuanto al estado civil 55 (83.3\%) refirió estar soltera, $5(7.6 \%)$ casada y $6(9.1 \%)$ en unión libre. Situación Laboral: 18 (27.3\%) trabajaba y 48 (72.7\%) no. Religión: 31 (47.0\%) afirmaron pertenecer a la religión católica. Ver Gráfica No 3.

Grafico $N^{\circ}$ 2: Áreas de Estudio de las Estudiantes Universitarias que Utilizan AOE

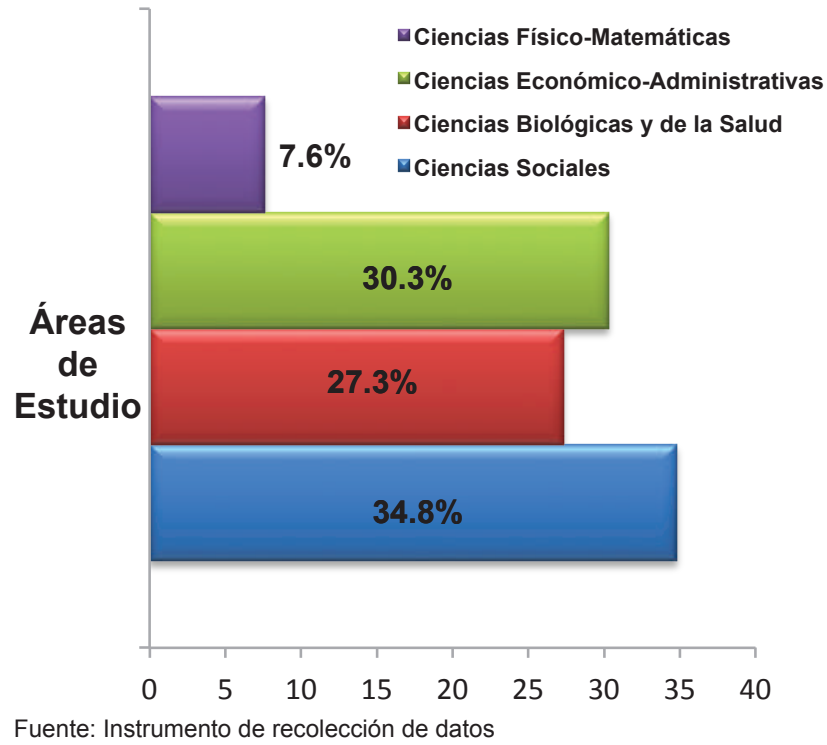

Gráfica $N^{\circ}$ 3: Religión a la que pertenecían las estudiantes

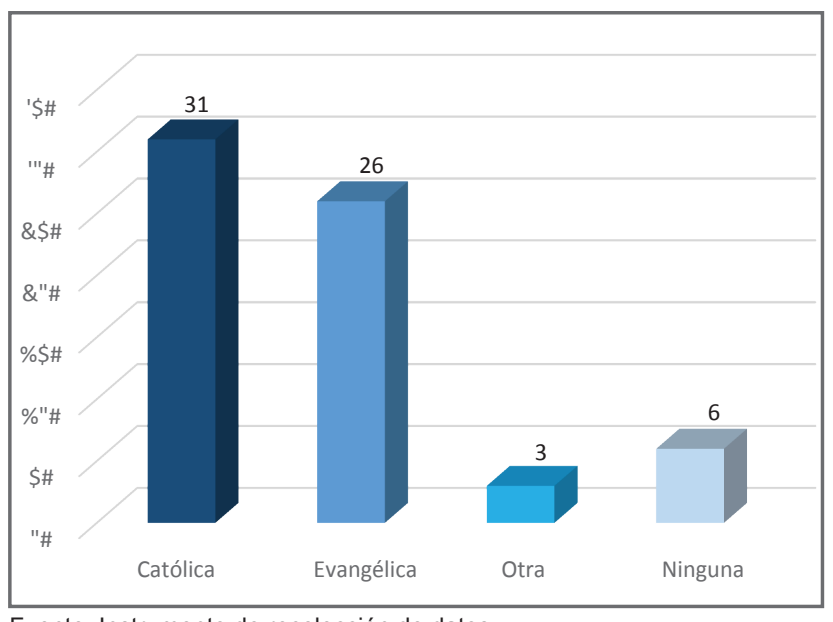

Fuente: Instrumento de recolección de datos

\section{DISCUSIÓN}

Respecto al nivel de conocimiento según este estudio el $12.1 \%$ posee un nivel alto, en contraste con países como, Ecuador, Costa Rica (Universidad de Costa Rica) y México, que reportaron $67.5 \%, 53.9 \%$ y $30.3 \%$ respectivamente. ${ }^{(7-9)}$

En relación a las características sociodemográficas este estudió arrojó que el $50.0 \%$ de las estudiantes que utilizan los $A O E$ se encuentran en un rango de edad entre $21 \mathrm{y}$ 24 años lo que no difiere mucho de un estudio llevado a cabo en Costa Rica donde la mayor anticoncepción de emergencia $(63.3 \%)$ se da en mujeres entre los 19 y 23 años. ${ }^{(8)}$ Según el lugar de procedencia y residencia un estudio realizado en Cataluña indica que la utilización es similar tanto en mujeres que proceden y/o residen en el área rural como en la urbana, $33.0 \%$ y $36.0 \%$ respectivamente. ${ }^{(10)}$ En Ecuador las estudiantes con ingresos familiares promedio $(79.0 \%)$ son las que más utilizan los AOE al igual que este estudio con un $48.5 \%{ }^{(7)} \mathrm{EI}$ estado civil sigue el mismo patrón en todas 
las investigaciones (Costa Rica, Ecuador y este estudio) que coinciden en que las solteras, $96.7 \%, 64.1 \%$ y $83.3 \%$, son las que más utilizan los AOE. En cuanto al área de estudio en Costa Rica el $62.5 \%$ de las estudiantes que utilizan los AOE pertenece a la ciencias sociales y de la salud, lo que no difiere en este estudio donde el $62.1 \%$ pertenecen a estas áreas. ${ }^{(7,8)}$ La religión predominante en este estudio y en el de la Universidad de Cuenca es la católica con $47.0 \%$ y $58.9 \%$ respectivamente y en cuanto a la situación laboral el estudio de la Universidad Veracruzana arroja que el $68.8 \%$ de las estudiantes que utilizan los AOE no trabajan, similar al $72.7 \%$ que recabó este estudio. . $^{(7,1-11)}$

En conclusión la utilización de anticonceptivos orales de emergencia por las estudiantes universitarias es una práctica realizada principalmente por jóvenes de 21 a 24 años, solteras, que no trabajan, con un ingreso familiar entre Lps. 8,000-16,000, procedentes del área urbana, católicas y de las carreras de las ciencias sociales, biológicas y de la salud. Las que los utilizan sin tener un conocimiento adecuado acerca de estos.

Se recomienda realizar más investigaciones sobre esta temática que permitan profundizar de manera cualitativa en el estudio de otras variables que no se pueden medir cuantitativamente y que brindan información sobre aspectos sociales, culturales y ético-morales.

\section{AGRADECIMIENTO}

Dr. Carlos Fuentes

Por la asesoría metodológica brindada.

\section{BIBLIOGRAFÍA}

1. Organización Mundial de la Salud. El aborto en cifras. Ginebra; 2004. [acceso el 2 de Febrero del 2015]. Womens Link Worlwilde. Disponible en: http://www. womenslinkworldwide.org/pdf_pro grams/es_prog_rr_col_factsheets_ci fras.pdf.

2. Díaz S, Croxatto HB. Anticoncepción de Emergencia. En: Ginecología 3a edición. Pérez Sánchez A. Santiago, Chile: Editorial Mediterráneo; 2003. pp. 1067-1073.

3. Rodríguez Yunta Eduardo. El levonorgestrel y su mecanismo de acción. ARS Medica, Revista de estudios médicos
Humanístico. 6(6) [acceso el 20 de Mayo del 2014]. Disponible en: http:// escuela.med.puc.cl/publ/arsmedica/ arsmedica6/art05.html

4. Sociedad Peruana de Obstetricia y Ginecología Centro de Promoción de los Derechos Sexuales y Reproductivos Fondo de Población de las Naciones Unidas. Guía nacional de atención integral de la salud sexual y reproductiva. 2010 [acceso el 9 de febrero del 2014]. Disponble en: http://www.unfpa.org.pe publicaciones/publicacionesperu/ PROMSEX-SustentoNormativoAOE.pdf

5. Salazar Andrés. Anticonceptivos orales 
de Emergencia. España; 2009. [acceso el 7 de febrero del 2015]. Disponible en: http://www.unfpa.org.pe/publicacio nes/publicacionesperu/SPOG-AOEMirada-Cientifica.pdf.

6. Plazas Andrew, Barriento Mateo. Centro de Salud Cerro del Aire. Majadahonda. Madrid, España. [acceso el 9 de Febrero del 2015]. Disponible en: http://www. jano.es/ficheros/sumarios/1/67/ 1543/56/1v67n1543a13069384pdf001. pdf.

7. Gómez - Molina Elisa, Idrovo - Freire Verónica. Conocimiento y Uso de la Anticoncepción de Emergencia en estudiantes de la escuela de enfermería y tecnología médica de la facultad de Ciencias Médicas de la Universidad de Cuencas, 2009-2010 Disponible en: http://dspace.ucuenca.edu.ec/bits tream/123456789/3738/1/MED30.pdf.

8. Martinez-Perez Julia, Rios Daniela. Conocimiento y uso de la píldora contraceptiva de emergencia en estudiantes universitarias. 2012 Sptiembre-Diciembre. Disponible en: http://cdigital.uv.mx/ bitstream/123456789/29288/1/tesis9. pdf.
9. Carvajal José, Maroto Adriana. Conocimiento y opiniones sobre la Píldora Anticonceptiva de Emergencia (PAE) a nivel de estudiantes universitarias y usuarias de un Equipo Básico de Atención Integral en Salud (EBAIS). Disponible en: ccp.ucr.ac.cr/ac/usuarias_adc.doc https://www.google.hn/?gws_rd=cr,ss I\&ei=ndugU5_DFK_IsATFhoG4AQ\# $q=u s o+d e+$ pastillas + de+emergencia+ en+estudiantes+universitarios.

10. Ros Clara, Miret Marta, Rué Montse. Estudio descriptivo sobre el uso de la anticoncepción de emergencia en Cataluña: Comparación entre una zona rural y una urbana. Gac Sanit [revista en la Internet]. 2009 [citado 25 febrero 2015]; 23(6): 496-500. Disponible en: http:// scielo.isciii.es/scielo.php?script=sci_art text\&pid=S0213-91112009000600002\& Ing=es.

11. Leyva-López A, Chávez-Ayala R, Atienzo EE, Allen-Leigh B, Ramírez-Villalobos D, Yunes-Díaz E, et al. Anticoncepción de emergencia en estudiantes mexicanos. Salud Publica Mex. 2010; 52:156-164.[ acceso en Mayo 2014] Disponible en: http://www.scielo.org.mx/ scielo. php? pid=S0036-36342010000 200008\&script=sci_arttext. 